\title{
Zwei wertvolle neue Helfer im Sortiment
}

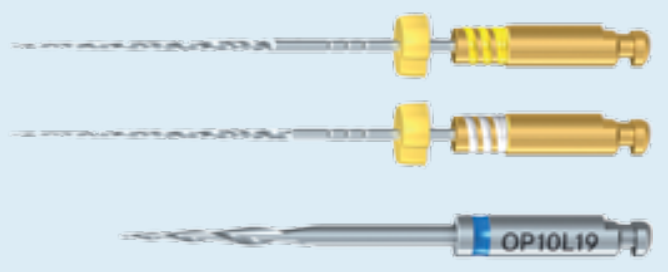

Komet baut sein Endodontie-Vollsortiment um 2 weitere Qualitätsprodukte aus - den Opener und den PathGlider. Bei der koronalen Erweiterung des Wurzelkanals arbeitet sich der Opener großzügig voran und befreit den Kanal bereits zu Anfang der Behandlung zuverlässig vom Großteil der Bakterien. Durch seinen Doppel-S-
Querschnitt zeigt das kurze Instrument eine angenehme Flexibilität, schützt vor Überpräparation und sorgt für einen schnellen und gründlichen Abtrag von koronal infiziertem Gewebe. Nach der primären Sondierung per Handfeile lässt sich anschließend der Gleitpfad mit dem PathGlider maschinell herstellen (erhältlich in
Länge 25, in den Größen 015 oder 020). Aufgrund seines Übergangstapers von .03 ebnet der PathGlider den nachfolgenden maschinellen Feilen (z. B. F360: Taper 04) einen leichten und sicheren Weg durch den Kanal. Durch die Flexibilität der Feile und ihrem maschinellen Antrieb reduziert sich zusätzlich das Risiko von Kanalverlagerungen und Stufenbildungen maßgeblich und trägt gleichzeitig zu einer deutlichen Zeiteinsparung bei.

Nach einer Pressemitteilung der Komet Dental Gebr. Brasseler GmbH \& Co KG, Lemgo

Internet: www.kometdental.de 